\title{
ASSOCIATED AND NON-ASSOCIATED FLOW RULES IN THE STRESS STATE PREDICTION
}

\author{
Vobejda R.*, Šebek F. **, Petruška J.***, Kubík P.****
}

\begin{abstract}
The paper deals with an application of the non-associated flow rule within a complex plasticity model. This leads to a significant improvement in the predicted stress state. The predicted stress state is closer to theoretical value on the contrary to the case when an associated flow rule - within quadratic or nonquadratic yield criteria - is used. The implementation of a more flexible plasticity model will be subject of a further study. The results suggest that the yield criterion has to be more versatile within HaighWestergaard space.
\end{abstract}

Keywords: Non-associated flow rule, Yield criterion, Deviatoric stress, Plain strain, Localization.

\section{Introduction}

A ductile fracture of metals is a phenomenon that has been researched for the many years as it finds the application in many fields of industry, like the automotive. The knowledge of the ductile failure process is very important especially in the field of forming operations. Such a technology can lead to the initiation of cracks in the material, which are not acceptable due to a reliability of the components. Nevertheless, the crack initiation may be predicted using the ductile criteria computationally. Such criteria depend on the stress state, usually represented by the stress triaxiality and normalized third invariant of deviatoric stress tensor (Šebek et al., 2018), but may be dependent on the strain rate or temperature as well. All these phenomena have to be studied experimentally, but with a heavy computational support, which allows to examine the state variables. These are observable experimentally, with the help of digital image correlation for example, but for sheet metals only, where the cracks initiate on the material surface. One of exceptions may be the torsion, where the crack initiates on the surface of a bulk material as well.

\section{Material}

The tensile test of flat grooved specimen was used within this study. Geometry with dimensions of the specimen are given in Fig. 1. The test was performed on the aluminium alloy 2024-T351. Zwick Z250 Allround-Line, tCII, and extensometer Zwick multiXtens were used for the plane strain testing under displacement control. The test speed of $1 \mathrm{~mm} / \mathrm{min}$ and gauge length of $22 \mathrm{~mm}$ were used.

\section{Plasticity models}

The specimen was modelled as one eighth due to the symmetry. It was discretized by C3D8R (8-node linear brick) elements with reduced integration, hourglass control and with the characteristic size of $0.075 \mathrm{~mm}$ across the width and $0.15 \mathrm{~mm}$ along the thickness. To speed up the computations, the semi-automatic mass scaling with the target time increment of $5 \cdot 10^{-7} \mathrm{~s}$ was employed.

* Ing. Radek Vobejda: Institute of Solid Mechanics, Mechatronics and Biomechanics, Faculty of Mechanical Engineering, Brno University of Technology, Technická 2896/2; 616 69, Brno; CZ, 170387@vutbr.cz

** Ing. František Šebek, PhD.: Institute of Solid Mechanics, Mechatronics and Biomechanics, Faculty of Mechanical Engineering, Brno University of Technology; Technická 2896/2; 616 69, Brno; CZ, sebek@fme.vutbr.cz

*** Prof. Ing. Jindřich Petruška, CSc.: Institute of Solid Mechanics, Mechatronics and Biomechanics, Faculty of Mechanical Engineering, Brno University of Technology, Technická 2896/2; 616 69, Brno; CZ, petruska@fme.vutbr.cz

**** Ing. Petr Kubík, PhD.: Institute of Solid Mechanics, Mechatronics and Biomechanics, Faculty of Mechanical Engineering, Brno University of Technology, Technická 2896/2; 616 69, Brno; CZ, kubik.p@fme.vutbr.cz 
Numerical computations were carried out within Abaqus/Explicit commercial finite element code. Three different plasticity models were used for the simulations of the experiment - the yield criteria with associated flow rule proposed by von Mises and Kroon and Faleskog (2013) and with non-associated flow rule proposed by Gao et al. (2011). All the models were implemented using Vectorised User MATerial.

At first, the plasticity model based on the associated flow rule was used. The numerical simulation of specimen at plane strain condition resulted in force response not corresponding to the experiment, when the quadratic yield criterion (von Mises) was used. In the case when the non-quadratic yield criterion (Kroon and Faleskog, 2013) was used for the numerical simulation, a correct force response was achieved. Nevertheless, normalized third invariant of deviatoric stress tensor did not match with the theoretical value for the plane strain condition, which is zero. This stress state was predicted correctly only when the quadratic yield criterion was used (Šebek et al., 2016). To perform a credible calibration of the ductile criterion, it is necessary to be able to obtain a correct force response as well as the values of the state variables. Therefore, the focus will be aimed at possibilities given by the utilization of non-associated flow rule (Gao et al., 2011; Šebek et al., 2019).

The associated or non-associated flow rules may be used to describe the behaviour of the material. The associated flow rule means that the flow rule is associated with the yield criterion and it is derived from a maximum dissipation postulate. Based on the associated flow rule, the plastic strain increment is normal to the yield surface. However, this principle cannot describe the behaviour of all materials. The direction of the real plastic strain increment may differ from the normal one to the yield surface. In that case, it is possible to use a non-associated flow rule, which introduces a scalar function named the plastic potential. This function does not have to be expressed identically with the yield function. Nevertheless, it usually differs from the yield function only by the material parameters in the most cases. The direction of the plastic strain increment is then derived from the function, which expresses the plastic potential. In the practice, the value of the plastic potential is not important, but the gradient of this function is (as it gives the direction of the plastic stain increment).

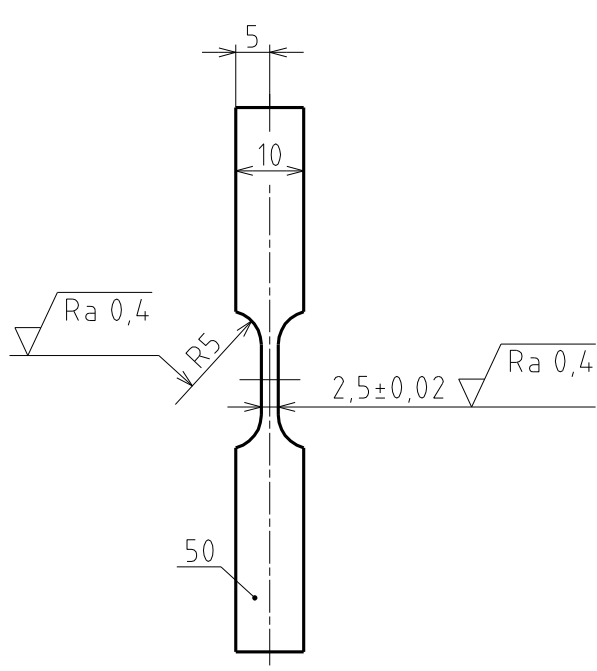

Fig. 1: Dimensions of the flat grooved specimen (all dimensions in $\mathrm{mm}$ ).

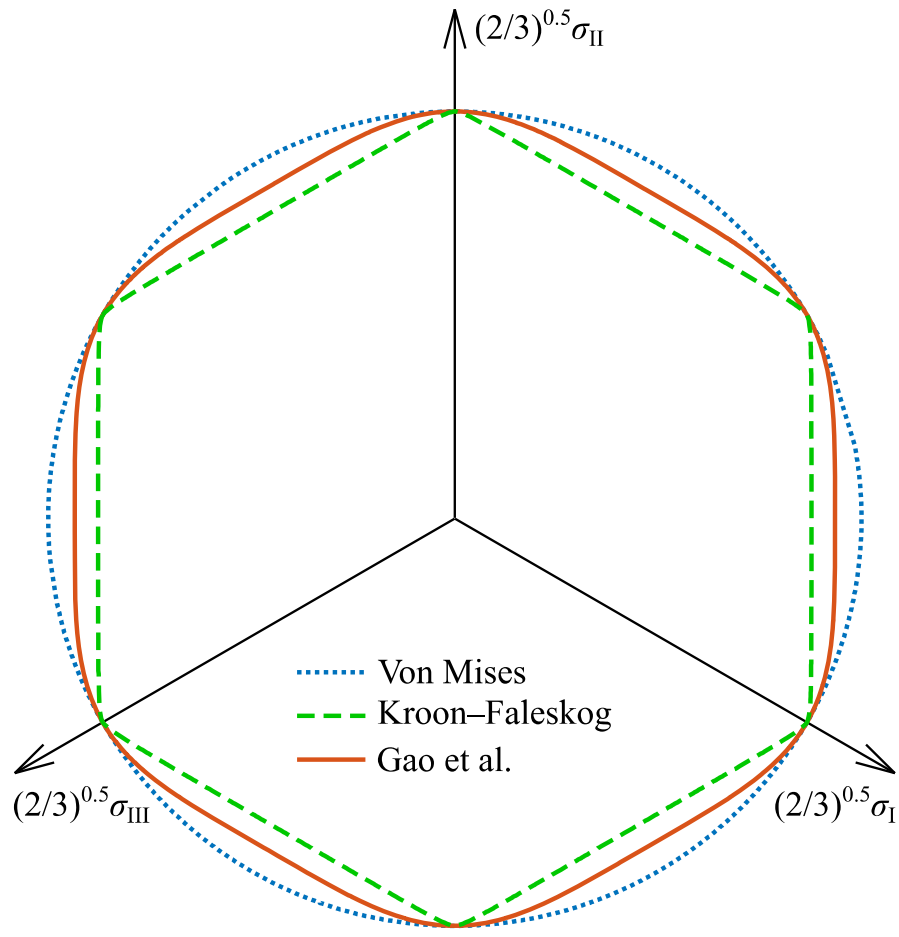

Fig. 2: Yield loci in the deviatoric plane.

Apart from von Mises and Kroon-Faleskog (Kroon and Faleskog, 2013) plasticity models that were taken from Šebek et al. (2016), Gao et al. plasticity model (Gao et al., 2011) that is described further in detail was considered in the present paper. The material parameters $a_{1}=a_{2}=b_{2}=0$ and $b_{1}=-60.75$ were used for yield function and plastic potential of Gao et al. plasticity model. Loci of all the three yield criteria are depicted in Fig. 2, where $\sigma_{k}$ for $k=I, I I, I I I$ are the principal stresses not order according to the magnitude. 
The non-quadratic yield function proposed by Gao et al. (2011) with non-associated flow rule is expressed using the first invariant of stress tensor $I_{1}$ and second and third invariants of the deviatoric stress tensor $J_{2}$ and $J_{3}$, respectively, as

$$
f_{y}=c_{1}\left(a_{1} I_{1}^{6}+27 J_{2}^{3}+b_{1} J_{3}^{2}\right)^{\frac{1}{6}}
$$

Where $a_{1}, b_{1}$ and $c_{1}$ are material parameters. Respective invariants may be expressed as

$$
\begin{gathered}
I_{1}=\sigma_{x x}+\sigma_{y y}+\sigma_{z z}, \\
J_{2}=\frac{1}{6}\left[\left(\sigma_{x x}-\sigma_{y y}\right)^{2}+\left(\sigma_{y y}-\sigma_{z z}\right)^{2}+\left(\sigma_{x x}-\sigma_{z z}\right)^{2}\right]+\sigma_{x y}^{2}+\sigma_{y z}^{2}+\sigma_{x z}^{2}, \\
J_{3}=\frac{1}{3}\left[\left(\sigma_{x y}^{2}-\sigma_{z z}^{2}\right)\left(\sigma_{x x}+\sigma_{y y}\right)+\left(\sigma_{y z}^{2}-\sigma_{x x}^{2}\right)\left(\sigma_{y y}+\sigma_{z z}\right)+\left(\sigma_{x z}^{2}-\sigma_{y y}^{2}\right)\left(\sigma_{x x}+\sigma_{z z}\right)\right] \\
+\frac{2}{27}\left(\sigma_{x x}+\sigma_{y y}+\sigma_{z z}\right)^{3}-\frac{2}{3}\left(\sigma_{x x} \sigma_{y z}^{2}+\sigma_{y y} \sigma_{x z}^{2}+\sigma_{z z} \sigma_{x y}^{2}\right)+2 \sigma_{x y} \sigma_{y z} \sigma_{x z}
\end{gathered}
$$

where $\sigma_{i j}$ for $i, j=x, y, z$ are the Cauchy stress tensor components. The material parameter $c 1$ can be found by considering the uniaxial tension condition, which results in

$$
c_{1}=\left(a_{1}+\frac{4}{729} b_{1}+1\right)^{-\frac{1}{6}} \text {. }
$$

The material parameter $c_{1}$ ensures that the value of the yield function is equal to the yield stress, when the material is subjected to the uniaxial stress. The plastic potential is expressed in the same manner. The only difference is the material parameters $a_{2}, b_{2}$ and $c_{2}$ as

$$
f_{p}=c_{2}\left(a_{2} I_{1}^{6}+27 J_{2}^{3}+b_{2} J_{3}^{2}\right)^{\frac{1}{6}} .
$$

Different shapes of the yield function (or the plastic potential eventually) are shown in Fig. 3. When the material parameters $a_{1}$ and $b_{1}$ are equal to zero, the yield function is identical with von Mises yield criterion. The following material parameter has to be $b_{1} \in\langle-60.75 ; 0\rangle$ in order to ensure that the yield function is convex. The yield function forms a dome when the material parameter is a positive number. This is probably unreal behaviour for most of the common constructional materials. Therefore, the material parameter was considered zero.
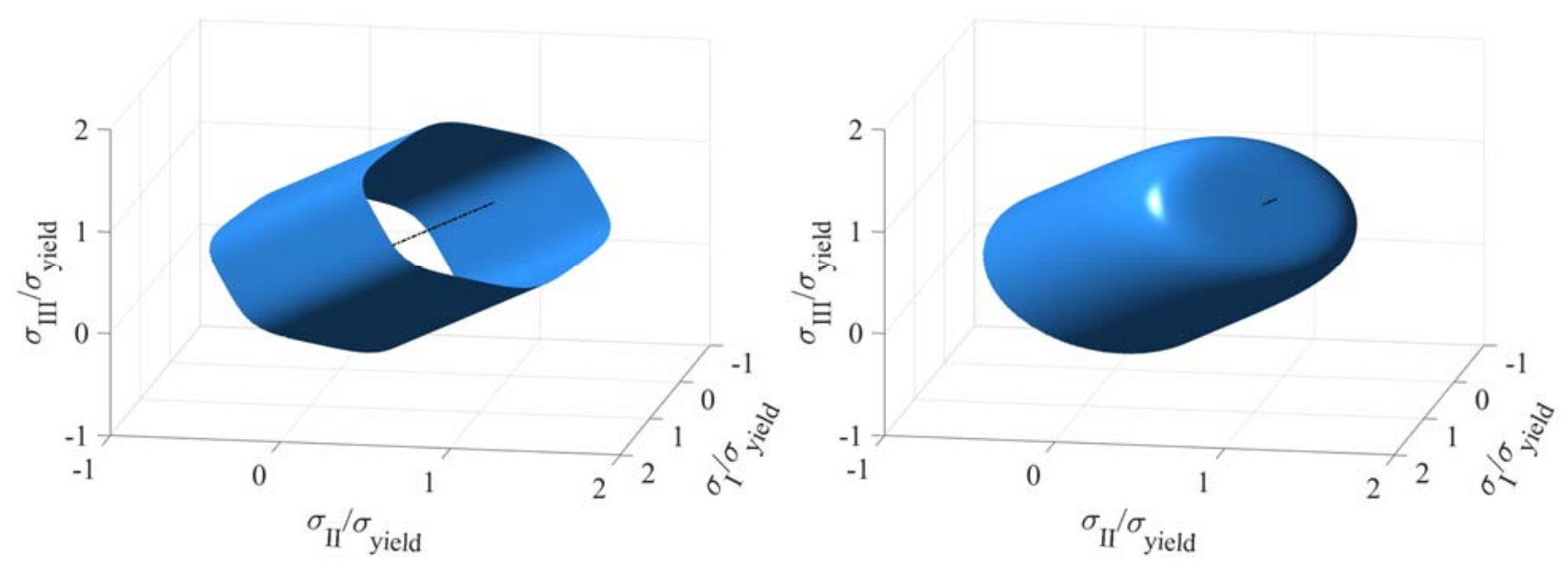

Fig. 3: Yield surface of Gao et al. plasticity model for the material parameters $a_{1}=0$ and $b_{1}=-60.75$ (left) and $a_{1}=0.001$ and $b_{1}=0$ (right). 


\section{Results and discussion}

There are force-displacement responses and evolutions of the normalized third invariant of deviatoric stress tensor for all the models of plasticity in Fig. 4. Von Mises yield criterion did not give a good agreement with the experimental force response, but the average value of the normalized third invariant of deviatoric stress tensor was approximately 0.34 , which is close to the theoretical value for plain strain that is zero. It is clear that Kroon-Faleskog yield criterion, which has an associated flow rule as well, corresponded better with experiments regarding the forces. On the other hand, it predicted incorrect value of the normalized third invariant of deviatoric stress tensor in the crack initiation location, which was in the centre of the specimen. The average value of the normalized third invariant of deviatoric stress tensor was approximately 0.94 approaching the uniaxial tension condition. Finally, the application of Gao et al. plasticity model resulted in worse force response when compared to Kroon-Faleskog plasticity model, but better than the one given by von Mises yield criterion. Moreover, the average value of the normalized third invariant of deviatoric stress tensor was approximately 0.31 , so even closer to zero than von Mises yield criterion.
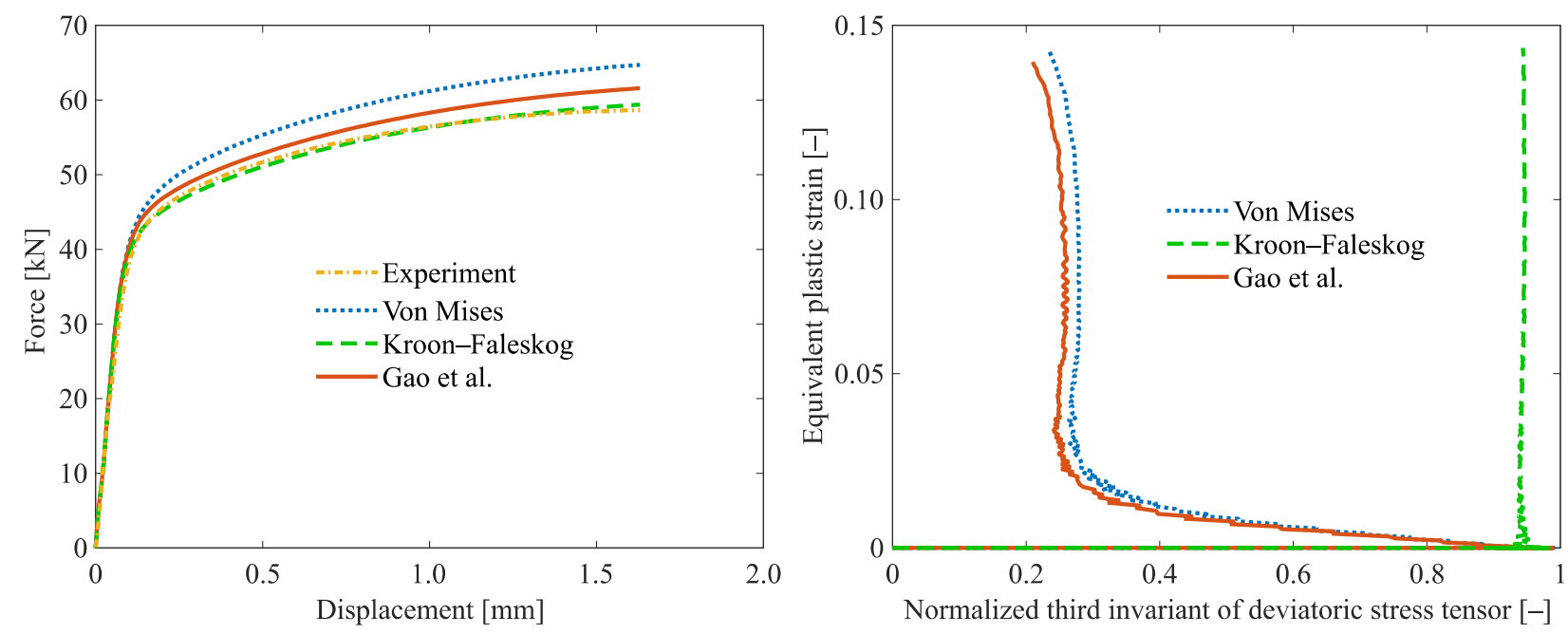

Fig. 4: Force-displacement responses (left) and evolutions of the normalized third invariant of deviatoric stress tensor (right).

\section{Conclusions}

It is evident from the results that the usage of the non-associated flow, proposed by Gao et al. (2011) here, could decisively influence the predictability of correct stress states, which are very important for the reliable ductile failure criteria calibration. The force-displacement response was not in the satisfying agreement with experiment for Gao et al. plasticity model, which revealed the limitations of the employed yield function. Therefore, the more flexible plasticity model will be sought in the future.

\section{Acknowledgement}

The work has been supported by the research project FV 20-35 at Faculty of Mechanical Engineering of Brno University of Technology.

\section{References}

Šebek, F., Petruška, J. and Kubík, P. (2018) Lode dependent plasticity coupled with nonlinear damage accumulation for ductile fracture of aluminium alloy. Materials \& Design, 137, pp. 90-107.

Kroon, M. and Faleskog, J. (2013) Numerical implementation of $J_{2}$ - and $J_{3}$-dependent plasticity model based on a spectral decomposition of the stress deviator. Computational Mechanics, 52, 5, pp. 1059-1070.

Šebek, F., Petruška, J. and Kubík, P. (2016) Behavior of Lode dependent plasticity at plane strain condition and its implication to ductile fracture. Solid State Phenomena, 258, pp. 213-216.

Šebek, F., Kubík, P., Petruška, J. and Zapletal, J. (2019) Non-associated flow rule in the prediction of complex stress states and deformations. In: Experimental Stress Analysis 2019, pp. 488-492.

Gao, X., Zhang T., Zhou J., Graham S. M., Hayden M. and Roe C. (2011) On stress-state dependent plasticity modeling: Significance of the hydrostatic stress, the third invariant of stress deviator and the non-associated flow rule. International Journal of Plasticity, 27, 2, pp. 217-231. 